\title{
Measurement of Rhodamine B absorption in self-assembled silica microwires using a Tablet as the optical source
}

John Canning, Masood Naqshbandi, Maxwell J. Crossley

John Canning, Masood Naqshbandi, Maxwell J. Crossley, "Measurement of Rhodamine B absorption in self-assembled silica microwires using a Tablet as the optical source," Proc. SPIE 8351, Third Asia Pacific Optical Sensors Conference, 83512E (31 January 2012); doi: 10.1117/12.914223

SPIE. Event: Asia Pacific Optical Sensors Conference, 2012, Sydney, Australia 


\title{
Measurement of Rhodamine B absorption in self-assembled silica microwires using a Tablet as the optical source
}

\author{
John Canning* ${ }^{\text {a,b }}$, Masood Naqshbandi ${ }^{\mathrm{a}, \mathrm{b}}$, and Maxwell J. Crossley ${ }^{\mathrm{b}}$ \\ ${ }^{a}$ Interdisciplinary Photonic Laboratories (iPL), School of Chemistry, 222 Madsen Building F09, The \\ University of Sydney, NSW 2006, Australia; \\ ${ }^{\mathrm{b}}$ School of Chemistry, The University of Sydney, NSW 2006, Australia \\ *john.canning@sydney.edu.au
}

\begin{abstract}
A simple demonstration utilising the optical light source of an Android tablet to obtain the absorption spectra of Rhodamine dye stained self-assembled silica microwires is demonstrated. The spectrum is collected using a portable Spectrometer. This highlights the potential of tablet technology as portable optical hardware in its own right and we discuss how to potentially achieve complete integration of spectrometer onto the tablet.
\end{abstract}

Keywords: Smartphones, optical hardware, Android, tablets, spectrometers, NBN, SmartGRid, biomedical sensors, sensing, optical sensors

\section{INTRODUCTION}

In recent work we reported the use of Android based Smartphones as potential optical hardware, where the active matrix organic light emitting diodes (OLED) potentially provide suitable excitation sources for basic fluorescence measurements [1]. The importance of this approach is that it potentially allows a new breed of low cost instruments for onsite measurements in the field where conventional technology cannot operate. For example, in certain regions of the world medical diagnostics is limited by available power supplies yet most villages have a local shop that power up mobile phones. Further, the flexible software that can control in any manner one chooses all the equipment of a smart phone based on object oriented Java means designer applets can be made readily available. Equally important, the combined hardware and wireless technology means data can be streamlined directly to laboratories for more detailed examination if necessary or data can be accessed for direct comparison of readings, a critical extension of the so-called medical SmartGrid which is often employed to justify the current Australian NBN deployment. The advent of tablet technology presents an additional tool in the armoury of potential chemical and biophotonics sensing technologies. Here, we demonstrate how a readily available tablet, using the Android honeycomb platform, can be similarly exploited to characterise the absorption of self-assembled silica wires doped with rhodamine B. This work is supported by very recent reports demonstrating that mobile phone CMOS sensors on smartphone cameras are suitable or a number of optical measurements, including chemiluminescence [2] and fluorescence [3], where excitation was performed using external LEDs - the use of these external sources can be potentially avoided by better exploiting continually improving OLED

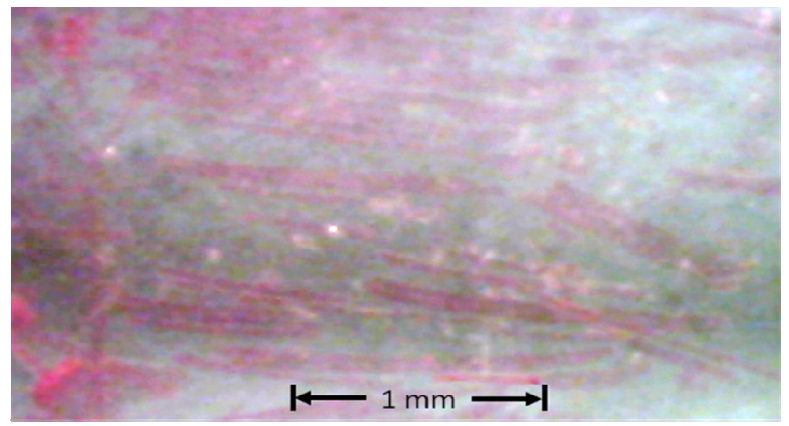

Figure 1. An image showing some of the doped silica wires formed after solvent evaporation on a standard glass slide. The bright pink colouring demonstrates successful staining with Rhodamine B.

Third Asia Pacific Optical Sensors Conference, edited by John Canning, Gangding Peng,

Proc. of SPIE Vol. 8351, 83512E - (c) 2012 SPIE · CCC code: 0277-786X/12/\$18 · doi: 10.1117/12.914223 
and liquid crystal technologies [1].

Silica nano and microwires are increasingly important in a number of applications including current sensors [4], chemical sensors [5] and evanescent field sensors [6]. The cold preparation of self-assembled structures based on nanoparticles generally, but especially silica $[7,8]$, opens up a new approach to the integration of materials that may otherwise be impossible to incorporate at higher temperatures or in the bulk. A clear illustration was our recent report on the incorporation of graphite into self-assembled silica mesostructured spheres where the presence of the graphite actually helped reduce the mechanical potential built up in the silica-only mesostructure as a result of very tight packing drive by van der Waals forces [7]. In this work, the advent of self-assembled silica wires, reported in more detail in [8] provides an unprecedented opportunity of integrating high concentration levels of many materials into these wires, including organic dyes such as Rhodamine. Thus, in this work we demonstrate this is so by measuring the absorption of a Rhodamine B doped self-assembled silica wire.

\section{PREPARATION OF SELF-ASSEMBLED SILICA WIRES}

We report in detail the preparation and the self-assembly of the silica microwires in [8], which includes the use of silica nanoparticles in low concentration $(5 \% \mathrm{w} / \mathrm{w})$ in water doped with solid state Rhodamine and ultra-sonicated for 10 minutes. The microwires are then self-assembled on a glass slide under atmospheric conditions $(1 \mathrm{~atm}, 273 \mathrm{~K})$ via evaporative self-assembly.

Figure 1 shows an optical image, taken using a low cost CMOS USB microscope, of some of the wires which precipitate during evaporation of the solvent onto a standard soda-lime glass slide. The wires in this experiment are a few millimeters in length with widths of a few tens of microns or more. They appear to be rectangular structures consistent with other measurements [8]. The obvious pink colouration is consistent with a high concentration of integrated Rhodamine $\mathrm{B}$, although are unable to quantify this easily. The overall wire distribution is in one plane only and the count relatively low.

\section{SPECTROSCOPY SETUP AND ABSORPTION MEASUREMENT}

The tablet used is an ASUS ee-PAD Transformer. Because of the larger screen (10.1"), the light emitting sources are presently not the leading industry active matrix organic LEDs (AMOLED) found in the next generation of Android Smartphones but rather a LED Backlight liquid crystal display. The Android 3.0 Honeycomb system means the same applet as that used previously [1] can be used to drive the individual RGB emitters - by turning them all on, the screen

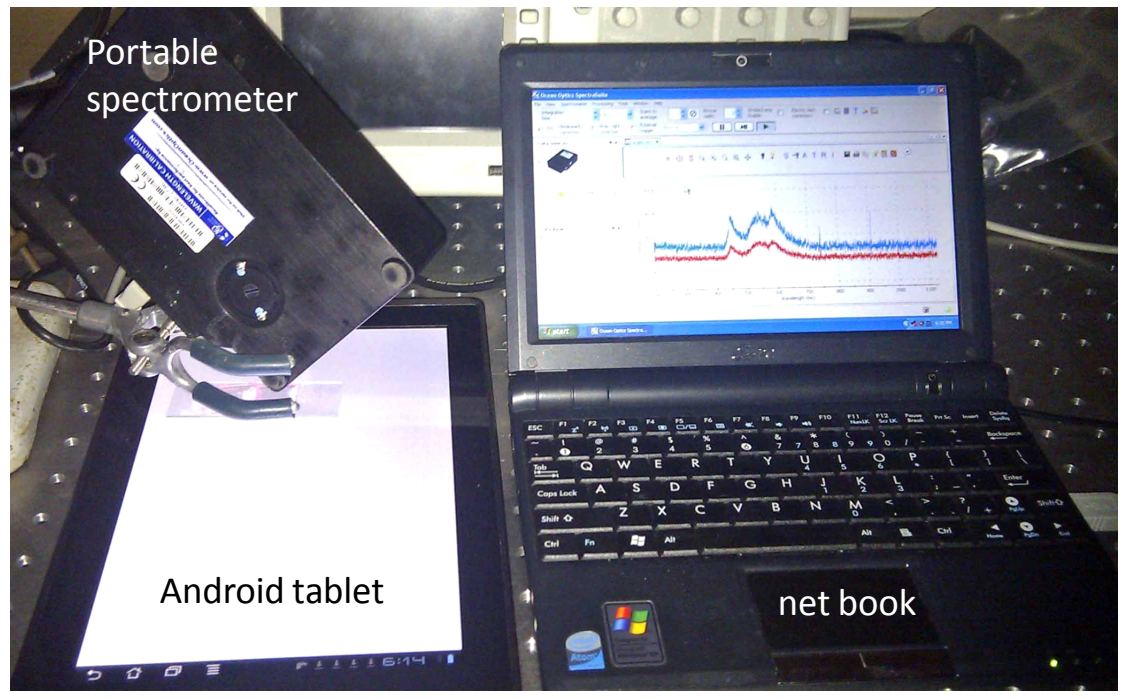

Figure 2. Experimental setup demonstrating a portable and readily assembled spectrometer with the Android tablet as the optical source. The sample with the self-assembled silica wires is placed on top of the screen just below a suspended portable spectrometer, driven by the netbook to the right. The netbook screen shows the raw data measured by the spectrometer with and without the sample. 

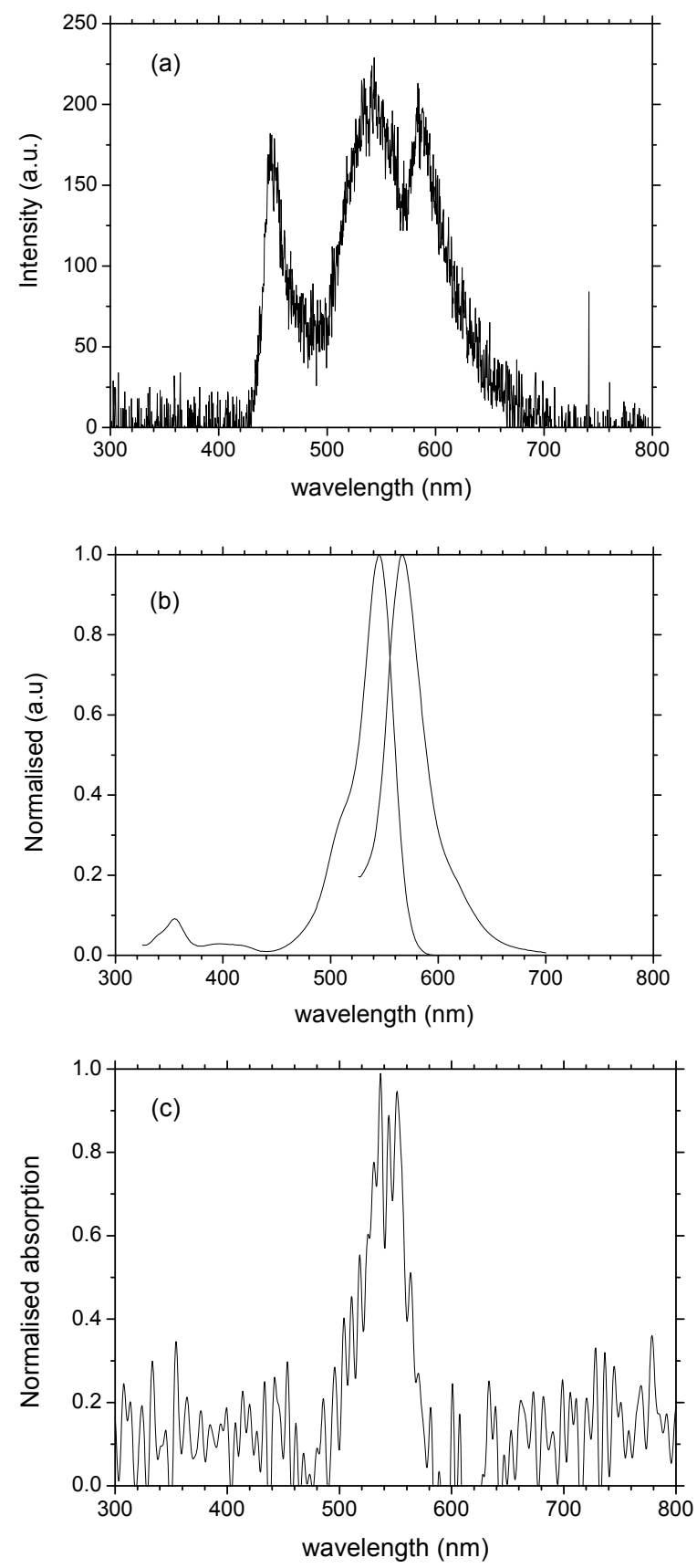

Figure 3. (a) RGB spectrum from the Tablet screen, (b) standard reference spectrum of Rhodamine B absorption and emission spectra in methanol and (c) measured Rhodamine B absorption within the Rhodamine-doped self-assembled silica microwires precipitated from water.

glows white as shown in Figure 2. The spectral profile was measured directly by placing in close proximity the fibre entry port of a portable Ocean Optics Spectrometer (also shown in Figure 2). A spectrum of the screen, taken without the sample, is shown in Figure 3 (a). From this, a maximum signal is observed around $550 \mathrm{~nm}$, which overlaps close to the peak excitation of rhodamine B, shown in Fig. 3 (b) for a standard reference sample in methanol. 
The sample shown in Fig. 1 was placed onto the screen, which can also be seen in Fig.2. The computer screen shown in Fig. 2 illustrates both the measured spectra with and without sample. By subtracting the two, the absorption of the sample can be obtained. The portable spectrometer had insufficient sensitivity and signal-to-noise to detect any fluorescence. Figure 3 (c) shows the normalised absorption measured from the sample without using any optical fibre input or optical lens, indicating that much better results could be obtained using a spectrometer that is optimised for bulk signal collection. Despite this, the absorption spectrum is clearly detected.

In spite of the apparent high concentrations suggested by the readily observed pink colouring of the wires, which may characterise aggregation and therefore potential coupled pathways for further absorption, the OLED emission is too weak to allow any significant additional one or two photon absorption from the excited state. The strong colouring can be attributed to just absorption of the green. This could be improved substantially by using a small collection lens at the input end that is matched to the designed spectrometer input NA. As such, using the approach described here we can make qualitative absorption measurements but not fluorescence in the current setup.

\section{DISCUSSION AND CONCLUSIONS}

The use of an Android tablet as an optical source has been demonstrated and the absorption of Rhodamine B dye doped into silica nanoparticles during the self-assembly of silica microwires has been measured. Despite the far from optimised configuration, the absorption was detected indicating, along with the strong coloration, that a high quantity of dye was integrated into the inorganic wires. These wires remain physically robust and can be stretched to in excess of 5\%. Thus, the silica microwire platform is potentially a low cost and useful method of producing sensors and biodiagnostics containing any particular chemical or bio sensor agent. Even if they are not directly attachable to the silica itself, the use of readily incorporated organics into the structure can potentially enable a multitude of functional methods to be adapted. This makes them an incredibly powerful sensor tool - they are capable of guiding light to further enhance resonant optical fields under appropriate conditions. Further, under appropriate conditions small wires on the nanoscale may offer an alternative to commonly deployed mesostructured spheres as a means to depositing markers, medicines and other labels into the body in medical applications.

With improved optimisation of the Smartphone and tablet systems described in this paper and our previous work, offer a simple low cost method of portable diagnostics for environmental monitoring and health and so on. More advanced systems might involve clip on tools (such as filters, prism, gratings and so on) to also take advantage of the photodetector potentially turning the technology into an all-in-one self-contained portable diagnostic instrument that can be charged up as per a normal mobile form. A total rethink of how this technology operates could be to design custom sensor units that are entirely based on the same protocols of Smartphones and platforms - this is entirely feasible given that equipment such as Ocean Optics is usually programmed in Java, the basis of Android. The advantage of the tablet over existing Smartphones is the real potential to be able to integrate fully all the netbook functionality, including software analysis, and instrument functionality entirely onto the tablet so that everything can be done simultaneously using the one screen the basic technology paradigm is now there and all that is required is custom software apps for each application. This will marks a new era in diagnostic capabilities that can be integrated directly through wireless into existing SmartGrid networks such as the Medical SmartGRid being touted as a driver of the Australian national broadband network (NBN).

Acknowledgements: This work was partially supported by Australian Research Council (ARC) DP grants A7248 and A7158. A private contribution from J. Canning for loaning the tablet and the idea of Smartphone, tablet sensors and Optical Sensor SmartGrids is acknowledged.

\section{REFERENCES}

[1] Canning, J., Lau, A., Naqshbandi, M., Petermann, I. and Crossley, M.J., "Measurement of fluorescence in a rhodamine-123 doped self-assembled "giant" mesostructured silica sphere using a Smartphone as optical hardware", Sensors, 11 (7), 7055-7062 (2011).

[2] Delaney, J.L., Hogan, C.F., Tian, J. and Shen, W., "Electrogenerated chemiluminescence in paper-based microfluidic sensors," Analyt. Chem., 83 (4), 1300-1306, (2011). 
[3] Zhu, H., Mavandadi, S., Coskun, A.F. and Yaglidere, A., "Optofluidic fluorescent imaging cytometry on a cellphone," Analyt. Chem., 87, 737-745, (2011).

[4] Belal, M., Song, Z., Jung, Y., Brambilla, G., and Newson, T.P., "Optical fiber microwire current sensor" Opt. Lett. 35, 3045-3047 (2010).

[5] Tan, W., Shi, Z.Y., Smith, S., Birnbaum, D., Kopelman, R., "Submicrometer intracellular chemical optical fiber sensors" Science 258, 778-781 (1992).

[6] Polynkin, P., Polynkin, A., Peyghambarian, N., and Mansuripur, M., "Evanescent field-based optical fiber sensing device for measuring the refractive index of liquids in microfluidic channels" Opt. Lett. 30, 1273-5 (2005).

[7] Naqshbandi, M., Canning, J., Lau, A. and Crossley, M.J., "Controlled Fabrication of Macroscopic Mesostructured Silica Spheres for Potential Diagnostics and Sensing Applications", The International Quantum Electronics Conference (IQEC)/The Conference on Lasers and Electro-Optics (CLEO) Pacific Rim, (IQEC/CLEO-Pacific Rim 2011), Sydney, Australia (2011)

[8] Naqshbandi, M., Canning, J., and Crossley, M.J., Submitted to $3^{\text {rd }}$ Asia Pacific optical Sensor Conference, Sydney, (2012). 